\title{
A New Estimation Study of the Stress-Strength Reliability for the Topp-Leone Distribution Using Advanced Sampling Methods
}

\author{
Abdullah M. Almarashi, ${ }^{1}$ Ali Algarni, ${ }^{1}$ Amal S. Hassan, ${ }^{2}$ M. Elgarhy $\left(\mathbb{D},{ }^{3}\right.$ Farrukh Jamal $\left(\mathbb{D}^{4},{ }^{4}\right.$ \\ Christophe Chesneau, ${ }^{5}$ Khudir Alrashidi, ${ }^{1}$ Wali Khan Mashwani $\left(0,{ }^{6}\right.$ and Heba F. Nagy ${ }^{2}$ \\ ${ }^{1}$ Faculty of Science, Department of Statistis, King Abdulaziz University, Jeddah, Saudi Arabia \\ ${ }^{2}$ Faculty of Graduate Studies for Statistical Research, Cairo University, Giza 12613, Egypt \\ ${ }^{3}$ The Higher Institute of Commercial Sciences, Al Mahalla Al Kubra 31951, Algarbia, Egypt \\ ${ }^{4}$ Department of Statistics, The Islamia University of Bahawalpur, Punjab 63100, Pakistan \\ ${ }^{5}$ Universit'e de Caen, LMNO, Campus II, Science 3, 14032, Caen, France \\ ${ }^{6}$ Institute of Numerical Sciences, Kohat University of Science \& Technology, Kohat 26000, Pakistan
}

Correspondence should be addressed to Wali Khan Mashwani; mashwanigr8@gmail.com

Received 8 September 2021; Accepted 4 October 2021; Published 28 October 2021

Academic Editor: Punit Gupta

Copyright ( 2021 Abdullah M. Almarashi et al. This is an open access article distributed under the Creative Commons Attribution License, which permits unrestricted use, distribution, and reproduction in any medium, provided the original work is properly cited.

\begin{abstract}
In this manuscript, we investigate the estimation of the unknown reliability measure $R=P[Y<X]$, in the case where $Y$ and $X$ are two independent random variables with Topp-Leone distributions. As the main contribution, various advanced sampling strategies are studied. The suggested strategies are simple random, ranked set, and median ranked set samplings. Firstly, based on the maximum likelihood, we give an efficient estimator of $R$ when the observations of the two random variables are selected from the same simple random sample. Secondly, such an estimator is addressed when the observations of the two random variables are selected from the ranked set sampling method. Then, based on median ranked set sampling, the maximum likelihood estimator of $R$ is addressed in all the four cases. When the observations from the two random variables are selected from the same set size, two cases are considered, while the other two cases are considered at different set sizes. A simulation research is developed to evaluate the behavior of the obtained estimates based on standard and median ranked set samplings with their simple random sampling equivalents. The ratio of mean square error is used to assess the effectiveness of these estimates.
\end{abstract}

\section{Introduction}

First and foremost, the simplified version of the Topp-Leone (TL) distribution is a bounded support one-parameter J-shaped distribution. It possesses a bathtub-shaped hazard rate (see [1]). For these reasons, it is very effective for modeling life-time tests. Mathematically, it is defined by a one-parameter probability density function (PDF) and a one-parameter cumulative distribution function (CDF) given, respectively, by

$$
\begin{aligned}
& f(x)=2 \alpha x^{\alpha-1}(1-x)(2-x)^{\alpha-1}, 0<x<1, \alpha>0, \\
& F(x)=x^{\alpha}(2-x)^{\alpha}, 0<x<1, \alpha>0 .
\end{aligned}
$$

Hereafter, the TL distribution will be sometimes denoted by $\operatorname{TL}(\alpha)$ in order to highlight the parameter.

In recent years, the TL distribution and its model have obtained a lot of attention in the literature. For instance, the authors in [2] proposed an extension of the TL distribution. The authors in [3] examined several features of the TL distribution, including the failure rate (classical and reversed) and the mean residual life time. A bivariate generalization of the TL distribution was introduced in [4]. Two-side based version of the TL distribution was proposed in [5]. Estimation of the stress-strength (SS) model was discussed based on censored samples in [6]. The author in [7] derived some moments properties of order statistics 
associated to the TL distribution. Using record values, the authors in [8] discussed Bayesian and non-Bayesian estimation methods for the unique parameter of the TL distribution. The author in [9] examined admissible minimax estimates of the TL distribution.

On the other hand, the notion of ranked set sampling (RSS) has been presented in [10] as a sampling scheme for data collection. This scheme is a useful technique when it is hard to measure a large number of elements, but it is easier visually (without inspection) to rank some of them. The RSS scheme has evolved into a complex strategy for enhancing mean estimation accuracy. The mathematical foundations for RSS design have been provided in $[11,12]$. The RSS scheme has been effective in applied sciences, with impressive wins in agriculture and ecology. The selection of a RSS of size $n$ entails the drawing of $n$ random samples, each containing $n$ units. Judgment is used to rank the $n$ units in each sample. The first sample is used to measure the smallest rank unit, and the second sample is used to measure the second smallest rank unit, and so on. When the unit with rank $n$ is chosen from the nth set, the first cycle is finished. It is worth noting that the $n$ observations are dispersed randomly. Because $n$ in the RSS is usually low, the following approach is used to create a sample of size (ns) by repeating its times or cycles.

Reference [13] suggested the median ranked set sampling (MRSS) scheme as another contribution to the RSS scheme. The MRSS method is easy to apply since only the middle of the sample is considered. The following is an outline of the MRSS method: We choose $n^{2}$ units randomly by using the simple random sampling (SRS) from the population, the $n^{2}$ randomly chosen units are divided into $n$ sets, each of size $n$, and the units within each set are ranked according to a variable of interest. If the set size $n$ is odd, the $((n+1) / 2)$ th lowest ranked unit in each set, which is the set's median, should be chosen for measurement. Furthermore, if $n$ is even, the $(n / 2)$ th smallest lowest ranked units from the first $n / 2$ sets should be chosen, followed by the $((n+2) / 2)$ th lowest ranked units from the second $n / 2$ sets. To obtain ns units, the cycle can be repeated $s$ times.

For several years, the SS models have attracted the attention of significant statisticians due to their applicability in distinct fields like economics, engineering, medicine, and quality control. To depict the life of a component with unpredictable strength and random stress, the SS model is utilized. The chance that the system will be able to sustain the stress placed on it is the reliability of the system, denoted by $R$. That is, $R=P(Y<X)$, where $X$ and $Y$ are continuous random variables (RVs) that are independent, modeling the random strength and stress, respectively. Applications of the SS model in medicine and engineering were provided in [14]. An outstanding review of the growth of the SS model up to 2003 was conducted in [15].

The problem of estimating $R$ has been intensively used in the statistical literature under various sampling schemes. In this work, we give a review of RSS schemes; for instance, the authors in $[16,17]$ concerned with estimating $R$ when $X$ and $Y$ are independent RVs with exponential distributions under the RSS. The authors in $[18,19]$ handled with the same problem but RR with Burr type XII distributions under some forms of RSS schemes. Also, the authors in [20] discussed the estimation of $R$ when $X$ and $Y$ are independent RVs with Weibull distributions under some versions of RSS. In this setting, Lindley distributions were supposed in [21], and exponentiated Pareto distributions were discussed in [22], both based on RSS. Using MRSS, the authors in [23] considered this estimation problem with generalized inverse exponential distributions.

Although no author has yet examined the estimation of $R$ for the TL model via ranking schemes and their modifications in the literature, we would like to point out that reference [6] contributed to this problem using censored schemes. So, in this manuscript, we use two different ranking schemes, namely, MRSS and RSS, as well as the traditional complete scheme, i.e., SRS, to converge on the estimation of $R$ when $X \sim \mathrm{TL}(\alpha)$ and $\mathrm{Y} \sim \mathrm{TL}(\beta)$ are independent. When both $X$ and $Y$ are selected from the same sample schemes (SRS, RSS, and MRSS), we get the ML estimator of R. Also, we obtain the ML of $R$ when both $X$ and $Y$ are selected from various sampling schemes (MRSS with odd size or even sample size). To demonstrate the theoretical results, a simulation study is provided. The following is a summary of the manuscript. When the SRS is considered, Section 2 calculates the ML estimator of R. When the RSS is considered, the ML estimator of $R$ is used in Section 3. When observed data from both $X$ and $Y$ are chosen from the MRSS with an even set size (MRSSE) or MRSS with an odd set size (MRSSO) or vice versa, Section 4 deals with the ML estimator of R. In Section 5, the simulation findings are discussed. In the last section, we wrap up the paper.

\section{ML Estimation of $\boldsymbol{R}$ Based on SRS}

Let $X \sim \mathrm{TL}(\alpha)$ and $Y \sim \mathrm{TL}(\beta)$ be independent. Then, with a standard integral development, the measure $R$ of the SS model for the TL distribution is basically given by

$$
R=P[Y<X]=2 \alpha \int_{0}^{1} x^{\alpha-1}(1-x)(2-x)^{\alpha-1} x^{\beta}(2-x)^{\beta} \mathrm{d} x=\frac{\alpha}{\alpha+\beta} .
$$

To get the ML estimator of $R$, we need to get the ML estimators for the unknown distribution parameters first. In this regard, suppose that $X_{1}, X_{2}, \ldots, X_{n^{*}}$ is a classical random sample from the distribution TL $(\alpha)$ and $Y_{1}, Y_{2}, \ldots, Y_{m^{*}}$ is a classical random sample from the distribution TL $(\beta)$. The related observations are denoted as small $x_{1}, x_{2}, \ldots, x_{n^{*}}$ and $y_{1}, y_{2}, \ldots, y_{m^{*}}$, and this is consistent across all the paper. The joint likelihood function for the observed samples is as follows: 
Scientific Programming

3

$$
\ell=(2 \alpha)^{n^{*}}(2 \beta)^{m^{*}} \prod_{i=1}^{n^{*}} x_{i}^{\alpha-1}\left(1-x_{i}\right)\left(2-x_{i}\right)^{\alpha-1} \prod_{j=1}^{m^{*}} y_{j}^{\beta-1}\left(1-y_{j}\right)\left(2-y_{j}\right)^{\beta-1}
$$

The corresponding joint log likelihood (LL) function is obtained as follows:

$$
\begin{aligned}
\ln \ell= & 2\left(\ln n^{*}\right)+2\left(\ln m^{*}\right)+n^{*} \ln \alpha+m^{*} \ln \beta+(\alpha-1) \sum_{i=1}^{n^{*}} \ln \left[x_{i}\left(2-x_{i}\right)\right] \\
& +\sum_{i=1}^{n^{*}} \ln \left(1-x_{i}\right)+(\beta-1) \sum_{j=1}^{m^{*}} \ln \left[y_{j}\left(2-y_{j}\right)\right]+\sum_{j=1}^{m^{*}} \ln \left(1-y_{j}\right) .
\end{aligned}
$$

As usual, the ML estimators of $\alpha$ and $\beta$ are produced by the maximization of LL with respect to the parameters, which is equivalent to differentiating the function in equation (4) and equating with zero, and we obtain

$$
\begin{aligned}
& \widehat{\alpha}=\frac{-n^{*}}{\sum_{i=1}^{n^{*}} \ln \left[x_{i}\left(2-x_{i}\right)\right]}, \\
& \widehat{\beta}=\frac{-m^{*}}{\sum_{j=1}^{m^{*}} \ln \left[y_{j}\left(2-y_{j}\right)\right]} .
\end{aligned}
$$

Thanks to the so-called invariance property, we deduce the ML estimator of $R$, denoted by $\widehat{R}$; it is derived by directly substituting equation (5) in equation (2).
3. ML Estimation of $\boldsymbol{R}$ Based on RSS

Suppose that $\left\{X_{i(i) e}, i=1,2, \ldots, n ; e=1,2, \ldots s_{x}\right)$ is a RSS observed from the distribution $\operatorname{TL}(\alpha)$, with sample size $n^{*}=$ $n s_{x}$, where $n$ is the set size and $s_{x}$ is the number of cycles, and that $\left\{Y_{j(j) h}, j=1,2 \ldots, m ; h=1,2, \ldots s_{y}\right)$ is a RSS observed from the distribution TL $(\beta)$, with sample size $m^{*}=m s_{y}$, where $m$ is the set size and $s_{y}$ is the number of cycles. In this setting, the following is the likelihood function $\ell_{1}$ of the observed samples:

$$
\begin{aligned}
\ell_{1} & =\prod_{e=1}^{s_{x}} \prod_{i=1}^{n} f_{i}\left(x_{i(i) e}\right) \prod_{h=1}^{s_{y}} \prod_{j=1}^{m} f_{j}\left(y_{j(j) h}\right), \\
f_{i}\left(x_{i(i) e}\right) & =\frac{2 \alpha n ! x_{i(i) e}^{\alpha i-1}}{(i-1) !(n-i) !}\left(1-x_{i(i) e}\right)\left(2-x_{i(i) e}\right)^{\alpha i-1}\left(1-x_{x_{i(i) e}}^{\alpha}\left(2-x_{i(i) e}\right)^{\alpha}\right)^{n-i}, x_{i(i) e}>0, \\
f_{j}\left(y_{j(j) h}\right) & =\frac{2 \beta m ! y_{j(j) h}^{\beta j-1}}{(j-1) !(m-j) !}\left(1-y_{j(j) h}\right)\left(2-y_{j(j) h}\right)^{\beta j-1}\left(1-y_{j(j) h}^{\beta}\left(2-y_{j(j) h}\right)^{\beta}\right)^{m-j}, y_{j(j) h}>0 .
\end{aligned}
$$

The LL function of $\ell_{1}$ is as follows:

$$
\begin{aligned}
& \ln \ell_{1} \propto s_{x} n \ln \alpha+s_{y} m \ln \beta+\sum_{e=1}^{s_{x}} \sum_{i=1}^{n}(\alpha i-1) \ln \left[x_{i(i) e}\left(2-x_{i(i) e}\right)\right]+\sum_{e=1}^{s_{x}} \sum_{i=1}^{n} \ln \left(1-x_{i(i) e}\right) \\
& +\sum_{e=1}^{s_{x}} \sum_{i=1}^{n}(n-i) \ln \left(1-x_{i(i) e}^{\alpha}\left(2-x_{i(i) e}\right)^{\alpha}\right)+\sum_{h=1}^{s_{y}} \sum_{j=1}^{m}(\beta j-1) \ln \left[y_{j(j) h}\left(2-y_{j(j) h}\right)\right] \\
& +\sum_{h=1}^{s_{y}} \sum_{j=1}^{m} \ln \left(1-y_{j(j) h}\right)+\sum_{h=1}^{s_{y}} \sum_{j=1}^{m}(m-j) \ln \left(1-y_{j(j) h}^{\beta}\left(2-y_{j(j) h}\right)^{\beta}\right) .
\end{aligned}
$$


With regard to $\alpha$ and $\beta$, we have

$$
\begin{aligned}
& \frac{\partial \ln \ell_{1}}{\partial \alpha}=\frac{n^{*}}{\alpha}+\sum_{e=1}^{s_{x}} \sum_{i=1}^{n} i \ln \left[x_{i(i) e}\left(2-x_{i(i) e}\right)\right]-\sum_{e=1}^{s_{x}} \sum_{i=1}^{n} \frac{(n-i) \ln \left[x_{i(i) e}\left(2-x_{i(i) e}\right)\right]}{x_{i(i) e}^{-\alpha}\left(2-x_{i(i) e}\right)^{-\alpha}-1}, \\
& \frac{\partial \ln \ell_{1}}{\partial \beta}=\frac{m^{*}}{\beta}+\sum_{h=1}^{s_{y}} \sum_{j=1}^{m} j \ln \left[y_{j(j) h}\left(2-y_{j(j) h}\right)\right]-\sum_{h=1}^{s_{y}} \sum_{j=1}^{m} \frac{(m-j) \ln \left[y_{j(j) h}\left(2-y_{j(j) h}\right)\right]}{y_{j(j) h}^{-\beta}\left(2-y_{j(j) h}\right)^{-\beta}-1} .
\end{aligned}
$$

Because the system "Equations (8) and (9) equal to zero" is difficult to solve analytically, we use an iterative technique to evaluate the ML estimators. Then, owing to the invariance property, the ML estimator of $R$ follows by inserting these estimators into Equation (2).

\section{ML Estimation of $\boldsymbol{R}$ Based on MRSS}

In this section, we investigate the ML estimator of $R$ based on MRSS in four situations. In the first and second situations, we estimate $R=P\left(Y_{\text {MRSSO }}<X_{\mathrm{MRSSO}}\right)$ and $R=P\left(Y_{\mathrm{MRSSE}}<\right.$ $\left.X_{\text {MRSSE}}\right)$, i.e., when both stress and strength are of the same size. When both $X$ and $Y$ have different set sizes, we estimate $R$ in the third and fourth situations.

4.1. Estimation with an Odd Set Size. Suppose that $X_{i(\mathcal{g}) e}$ where $i=1, \ldots, n, e=1, \ldots, s_{x}$, and $g=[n+1 / 2]$ is the MRSS with sample size $n^{*}=n s_{x}$, where $n$ is the set size and $s_{x}$ is the number of cycles, selected from the distribution TL $(\alpha)$, and that $Y_{j(k) h}$ where $j=1, \ldots, m, h=1, \ldots, s_{y}$, and $k=[m+1 / 2]$ is the MRSS with sample size $m s_{y}$, where $m$ is the set size and $s_{y}$ is the number of cycles, selected from the distribution TL $(\beta)$. The associated likelihood function $\ell_{2}$ for the observed samples is given by

$$
\begin{aligned}
\ell_{2} & =\prod_{e=1}^{s_{x}} \prod_{i=1}^{n} f_{g}\left(x_{i(g) e}\right) \prod_{h=1}^{s_{y}} \prod_{j=1}^{m} f_{k}\left(y_{j(k) h}\right), \\
f_{g}\left(x_{i(g) e}\right) & =\frac{n ! 2 \alpha x_{i(g) e}^{\alpha g-1}}{[(g-1) !]^{2}}\left(1-x_{i(g) e}\right)\left(2-x_{i(g) e}\right)^{\alpha g-1}\left(1-x_{x_{i(g) e}}^{\alpha}\left(2-x_{i(g) e}\right)^{\alpha}\right)^{g-1}, x_{i(g) e}>0, \\
f_{k}\left(y_{j(k) h}\right) & =\frac{2 m ! \beta y_{j(k) h}^{\beta k-1}}{[(k-1) !]^{2}}\left(1-y_{j(k) h}\right)\left(2-y_{j(k) h}\right)^{\beta k-1}\left(1-y_{j(k) h}^{\beta}\left(2-y_{j(k) h}\right)^{\alpha}\right)^{k-1}, y_{j(k) h}>0 .
\end{aligned}
$$

The LL function of $\ell_{2}$ is as follows:

$$
\begin{aligned}
& \ln \ell_{2} \propto s_{x} n \ln \alpha+s_{y} m \ln \beta+\sum_{e=1}^{s_{x}} \sum_{i=1}^{n}(\alpha g-1) \ln \left[x_{i(g) e}\left(2-x_{i(g) e}\right)\right]+\sum_{e=1}^{s_{x}} \sum_{i=1}^{n} \ln \left(1-x_{i(g) e}\right), \\
& +\sum_{e=1}^{s_{x}} \sum_{i=1}^{n}(g-1) \ln \left(1-x_{i(g) e}^{\alpha}\left(2-x_{i(g) e}\right)^{\alpha}\right)+\sum_{h=1}^{s_{y}} \sum_{j=1}^{m}(\beta k-1) \ln \left[y_{j(k) h}\left(2-y_{j(k) h}\right)\right], \\
& +\sum_{h=1}^{s_{y}} \sum_{j=1}^{m} \ln \left(1-y_{j(k) h}\right)+\sum_{h=1}^{s_{y}} \sum_{j=1}^{m}(k-1) \ln \left(1-y_{j(k) h}^{\beta}\left(2-y_{j(k) h}\right)^{\beta}\right) .
\end{aligned}
$$

Again, the ML estimators of $\alpha$ and $\beta$ are derived by maximizing $\ln \ell_{2}$. In this regard, the first partial derivatives of $\ln \ell_{2}$ with respect to the parameters are 


$$
\begin{aligned}
& \frac{\partial \ln \ell_{2}}{\partial \alpha}=\frac{n^{*}}{\alpha}+\sum_{e=1}^{s_{x}} \sum_{i=1}^{n} g \ln \left[x_{i(g) e}\left(2-x_{i(g) e}\right)\right]-\sum_{e=1}^{s_{x}} \sum_{i=1}^{n} \frac{(g-1) \ln \left[x_{i(g) e}\left(2-x_{i(g) e}\right)\right]}{x_{i(g) e}^{-\alpha}\left(2-x_{i(g) e}\right)^{-\alpha}-1}, \\
& \frac{\partial \ln \ell_{2}}{\partial \beta}=\frac{m^{*}}{\beta}+\sum_{h=1}^{s_{y}} \sum_{j=1}^{m} k \ln \left[y_{j(k) h}\left(2-y_{j(k) h}\right)\right]-\sum_{h=1}^{s_{y}} \sum_{j=1}^{m} \frac{(k-1) \ln \left[y_{j(k) h}\left(2-y_{j(k) h}\right)\right]}{y_{j(k) h}^{-\beta}\left(2-y_{j(k) h}\right)^{-\beta}-1} .
\end{aligned}
$$

By solving the system "Equations (12) and (13) equal to zero," we get the ML estimators of $\alpha$ and $\beta$. Hence, reliability estimator of $R$ is produced by immediately by substitution of parameter's estimators in equation (2).

4.2. Estimation with an Even Set Size. Suppose $\left\{X_{i(q) e}\right.$, $\left.i=1, \ldots, q ; e=1, \ldots, s_{x}\right\} \cup\left\{X_{i(q+1) e}, i=q+1, \ldots, n ; e=1, \ldots, s_{x}\right\}$, with even set sizes where $q=n / 2$ be the observed MRSSE selected from the distribution TL $(\alpha)$. Also, suppose $\left\{Y_{j(\mathrm{v}) h}\right.$, $\left.j=1, \ldots, \mathrm{v} ; h=1, \ldots, s_{y}\right\} \cup\left\{Y_{j(\mathrm{v}+1) e}, j=v+1, \ldots, m ; h=1, \ldots, s_{y}\right\}$, with even set sizes where $v=m / 2$ be the observed MRSSE selected from the distribution TL $(\beta)$. Therefore, the likelihood function $\ell_{3}$ of the observed data is written as follows:

$$
\begin{aligned}
& \ell_{3}=\prod_{e=1}^{s_{x}} \prod_{i=1}^{q} f_{q}\left(x_{i(q) e}\right) \prod_{e=1}^{s_{x}} \prod_{i=q+1}^{n} f_{q+1}\left(x_{i(q+1) e}\right) \prod_{h=1}^{s_{y}} \prod_{j=1}^{v} f_{\nu}\left(y_{j(v) h}\right) \prod_{h=1}^{s_{y}} \prod_{j=\nu+1}^{m} f_{\nu+1}\left(y_{j(v+1) h}\right), \\
& f_{q}\left(x_{i(q) e}\right)=\frac{n ! 2 \alpha x_{i(q) e}^{\alpha q-1}}{(q-1) ! q !}\left(1-x_{i(q) e}\right)\left(2-x_{i(q) e}\right)^{\alpha q-1}\left(1-x_{x_{i(q) e}}^{\alpha}\left(2-x_{i(q) e}\right)^{\alpha}\right)^{q}, x_{i(q) e}>0, \\
& f_{q+1}\left(x_{i(q+1) e}\right)=\frac{n ! 2 \alpha x_{i(q+1) e}^{\alpha(q+1)-1}}{(q-1) ! q !}\left(1-x_{i(q+1) e}\right)\left(2-x_{i(q+1) e}\right)^{\alpha(q+1)-1}\left(1-x_{x_{i(q+1) e}}^{\alpha}\left(2-x_{i(q+1) e}\right)^{\alpha}\right)^{q-1}, x_{i(q+1) e}>0 \\
& f_{\nu}\left(\left(y_{j(v) h}\right)=\frac{m ! 2 \beta y_{j(\nu) h}^{\beta \nu-1}}{(\nu-1) ! v !}\left(1-y_{j(v) h}\right)\left(2-y_{j(v) h}\right)^{\beta \nu-1}\left(1-y_{j(v) h}^{\beta}\left(2-y_{j(v) h}\right)^{\beta}\right)^{\nu}, y_{j(v) h}>0,\right. \\
& f_{\nu+1}\left(y_{j(\nu+1) h}\right)=\frac{m ! 2 \beta y_{j(\nu+1) h}^{\beta(\nu+1)-1}}{(\nu-1) ! \nu !}\left(1-y_{j(\nu+1) h}\right)\left(2-y_{j(\nu+1) h}\right)^{\beta(\nu+1)-1}\left(1-y_{j(\nu+1) h}^{\beta}\left(2-y_{j(\nu+1) h}\right)^{\beta}\right)^{\nu-1}, y_{j(\nu+1) h}>0 .
\end{aligned}
$$

The LL function of $\ell_{3}$ for $\alpha$ and $\beta$ based on MRSSE is

$$
\begin{aligned}
& \ln e_{3} \propto \sum_{e=1}^{s_{x}} \sum_{i=1}^{q}\left[(\alpha q-1) \ln \left(x_{i(q) e} T_{1}\right)+\ln \left(1-x_{i(q) e}\right)\right]+\sum_{e=1}^{s_{x}} \sum_{i=q+1}^{n}(\alpha(q+1)-1) \ln \left(x_{i(q+1) e} T_{2}\right) \\
& \quad+s_{x} n \ln \alpha+s_{y} m \ln \beta+\sum_{e=1}^{s_{x}} \sum_{i=q+1}^{n}\left[\ln \left(1-x_{i(q+1) e}\right)+(q-1) \ln \left(1-x_{i(q+1) e}^{\alpha} T_{2}^{\alpha}\right)\right] \\
& \quad+\sum_{e=1}^{s_{x}} \sum_{i=1}^{q} q \ln \left(1-x_{i(q) e}^{\alpha} T_{1}^{\alpha}\right)+\sum_{h=1}^{s_{y}} \sum_{j=1}^{v}\left[(\beta \nu-1) \ln \left(y_{j(v) h} N_{1}\right)+\ln \left(1-y_{j(v) h}\right)\right] \\
& \quad+\sum_{h=1}^{s_{y}} \sum_{j=v+1}^{m}(\beta(\nu+1)-1) \ln \left(y_{j(v+1) h} N_{2}\right)+\sum_{h=1}^{s_{y}} \sum_{j=v+1}^{m}\left[\ln \left(1-y_{j(v+1) h}\right)+(v-1) \ln \left(1-y_{j(v+1) h}^{\beta} N_{2}^{\beta}\right)\right] \\
& +\sum_{h=1}^{s_{y}} \sum_{j=1}^{v} v \ln \left(1-y_{j(v) h}^{\beta} N_{2}^{\beta}\right),
\end{aligned}
$$


where $T_{1}=\left(2-x_{i(q) e}\right), T_{2}=\left(2-x_{i(q+1) e}\right), N_{1}=$ $\left(2-y_{j(v) h}\right)$, and $N_{2}=\left(2-y_{j(v+1) h}\right)$. Hence,

$$
\begin{aligned}
\frac{\partial \ln \ell_{3}}{\partial \alpha}= & \frac{n^{*}}{\alpha}+\sum_{e=1}^{s_{x}} \sum_{i=1}^{q} q \ln \left(x_{i(q) e} T_{1}\right)+\sum_{e=1}^{s_{x}} \sum_{i=q+1}^{n}(q+1) \ln \left(x_{i(q+1) e} T_{2}\right) \\
& -\sum_{e=1}^{s_{x}} \sum_{i=1}^{q} \frac{q \ln \left(x_{i(q) e} T_{1}\right)}{x_{i(q) e}^{-\alpha} T_{1}^{-\alpha}-1}+\sum_{e=1}^{s_{x}} \sum_{i=q+1}^{n} \frac{(q+1) \ln \left(x_{i(q+1) e} T_{2}\right)}{x_{i(q+1) e}^{-\alpha} T_{2}^{-\alpha}-1}, \\
\frac{\partial \ln \ell_{3}}{\partial \beta}= & \frac{m^{*}}{\beta}+\sum_{h=1}^{s_{y}} \sum_{j=1}^{v} \nu \ln \left(y_{j(v) h} N_{1}\right)+\sum_{h=1}^{s_{y}} \sum_{j=\nu+1}^{m}(\nu-1) \ln \left(y_{j(v+1) h} N_{2}\right) \\
& -\sum_{h=1}^{s_{y}} \sum_{j=1}^{v} \frac{\nu \ln \left(y_{j(v) h} N\right)_{1}}{y_{j(v) h}^{-\beta} N_{1}^{-\beta}}+\sum_{h=1}^{s_{y}} \sum_{j=\nu+1}^{m} \frac{(\nu-1) \ln \left(y_{j(v+1) h} N_{2}\right)}{y_{j(\nu+1) h}^{-\beta} N_{2}^{-\beta}-1} .
\end{aligned}
$$

We solve "Equations (16) and (17) equal to zero" to take out the ML estimators. Further, we insert the obtained ML estimators in Equation (2) to have an estimator of $R$.

4.3. Estimation with an Odd Strength and Even Stress Sizes. Here, we get the ML estimator of $R$ under the following configuration: based on the MRSSO, we select observed samples of $X$ from the distribution $\operatorname{TL}(\alpha)$; based on the MRSSE, we select observed samples of $Y$ from the distribution $\operatorname{TL}(\beta)$.
Suppose that $X_{i\left(g_{e}\right)}$, where $i=1, \ldots, n, e=1, \ldots, s_{x}$, $g=[(n+1) / 2]$, with sample size $n s_{x}$, where $n$ is the odd set size and $s_{x}$ is the number of cycles, is the observed MRSSO from the distribution $\operatorname{TL}(\alpha)$, and that $\left\{Y_{j(} v_{) h}, j=1, \ldots, v\right.$; $\left.h=1, \ldots, s_{y}\right\} \cup\left\{Y_{j(v+1) e}, j=v+1, \ldots, m ; h=1, \ldots, s_{y}\right\}$, with even set sizes where $v=m / 2$, is the observed MRSSE selected from the distribution $\operatorname{TL}(\beta)$. Therefore, the associated likelihood function $\ell_{4}$ is

$$
\ell_{4}=\prod_{e=1}^{s_{x}} \prod_{i=1}^{n} f_{g}\left(x_{i(g) e}\right) \prod_{h=1}^{s_{y}} \prod_{j=1}^{v} f_{v}\left(y_{j(v) h}\right) \prod_{h=1}^{s_{y}} \prod_{j=v+1}^{m} f_{\nu+1}\left(y_{j(v+1) h}\right),
$$

where the PDFs of $X_{i\left(g_{)},\right.}, Y_{j}\left(v_{)}\right)$and $Y_{j(v+1) h}$ are as defined before. The LL of the observed data is

$$
\begin{aligned}
& \ln \ell_{4} \propto s_{x} n \ln \alpha+s_{y} m \ln \beta+\sum_{e=1}^{s_{x}} \sum_{i=1}^{n}(\alpha g-1) \ln \left[x_{i(g) e}\left(2-x_{i(g) e}\right)\right]+\sum_{e=1}^{s_{x}} \sum_{i=1}^{n} \ln \left(1-x_{i(g) e}\right) \\
& \quad+\sum_{e=1}^{s_{x}} \sum_{i=1}^{n}(g-1) \ln \left(1-x_{i(g) e}^{\alpha}\left(2-x_{i(g) e}\right)^{\alpha}\right)+\sum_{h=1}^{s_{y}} \sum_{j=1}^{\nu}(\beta \nu-1) \ln \left(y_{j(v) h} N_{1}\right) \\
& \quad+\sum_{h=1}^{s_{y}} \sum_{j=1}^{v} \ln \left(1-y_{j(v) h}\right)+\sum_{h=1}^{s_{y}} \sum_{j=v+1}^{m}(\beta(v+1)-1) \ln \left(y_{j(v+1) h} N_{2}\right)+\sum_{h=1}^{s_{y}} \sum_{j=v+1}^{m} \ln \left(1-y_{j(v+1) h}\right) \\
& +\sum_{h=1}^{s_{y}} \sum_{j=1}^{v} v \ln \left(1-y_{j(v) h}^{\beta} N_{1}^{\beta}\right)+\sum_{h=1}^{s_{y}} \sum_{j=v+1}^{m}(\nu-1) \ln \left(1-y_{j(\nu+1) h}^{\beta} N_{2}^{\beta}\right) .
\end{aligned}
$$

Maximizing $\ln \ell_{4}$ with respect to $\alpha$ and $\beta$, we obtain the ML estimators. The partial derivatives of $\ln \ell_{4}$ with respect to the parameters are given by 


$$
\begin{aligned}
\frac{\partial \ln \ell_{4}}{\partial \alpha}= & \frac{n^{*}}{\alpha}+\sum_{e=1}^{s_{x}} \sum_{i=1}^{n} g \ln \left[x_{i(g) e}\left(2-x_{i(g) e}\right)\right]-\sum_{e=1}^{s_{x}} \sum_{i=1}^{n} \frac{(g-1) \ln \left[x_{i(g) e}\left(2-x_{i(g) e}\right)\right]}{x_{i(g) e}^{-\alpha}\left(2-x_{i(g) e}\right)^{-\alpha}-1}, \\
\frac{\partial \ln \ell_{4}}{\partial \beta}= & \frac{m^{*}}{\beta}+\sum_{h=1}^{s_{y}} \sum_{j=1}^{v} v \ln \left(y_{j(v) h} N_{1}\right)+\sum_{h=1}^{s_{y}} \sum_{j=v+1}^{m}(\nu+1) \ln \left(y_{j(v+1) h} N_{2}\right) \\
& -\sum_{h=1}^{s_{y}} \sum_{j=1}^{v} \frac{\nu \ln \left(y_{j(v) h} N_{1}\right)}{y_{j(v) h}^{-\beta} N_{1}^{-\beta}-1}+\sum_{h=1}^{s_{y}} \sum_{j=v+1}^{m} \frac{(\nu-1) \ln \left(y_{j(v+1) h} N_{2}\right)}{y_{j(v+1) h}^{-\beta} N_{2}^{-\beta}-1} .
\end{aligned}
$$

There appear to be no closed form solutions to Equations (20) and (21) when equal with zero. As a result, numerical techniques are used to find the solution.

4.4. Estimation with an Even Strength and Odd Stress Sizes. Here, the estimator of $R=P\left(Y_{\text {MRSSO }}<X_{\text {MRSSE }}\right)$ is derived, where $X$ is selected from the distribution TL $(\alpha)$ based on the MRSSE and $Y$ is selected from the distribution $\operatorname{TL}(\beta)$ based on the MRSSO. Let $\left\{X_{i(q) e}, i=1, \ldots, q ; e=1, \ldots, s_{x}\right\} \cup\left\{X_{i(q+1) e}\right.$, $\left.i=q+1, \ldots, n ; e=1, \ldots, s_{x}\right\}$, where $q=n / 2$ and $Y_{j(k) h}$ where $j=1, \ldots, m, d=1, \ldots, s_{y}$, and $h=[(m+1) / 2]$. The likelihood function $\ell_{5}$ of the observed data is given by

$$
\ell_{5}=\prod_{e=1}^{s_{x}} \prod_{i=1}^{q} f_{q}\left(x_{i(q) e}\right) \prod_{e=1}^{s_{x}} \prod_{i=q+1}^{n} f_{q+1}\left(x_{i(q+1) e}\right) \prod_{h=1}^{s_{y}} \prod_{j=1}^{m} f_{k}\left(y_{j(k) h}\right) .
$$

Therefore, the LL function of $\ell_{5}$ for observed data is

$$
\begin{aligned}
& \ln \ell_{5} \propto s_{x} n \ln \alpha+s_{y} m \ln \beta+\sum_{e=1}^{s_{x}} \sum_{i=1}^{q}\left[(\alpha q-1) \ln \left(x_{i(q) e} T_{1}\right)+\ln \left(1-x_{i(q) e}\right)\right]+\sum_{e=1}^{s_{x}} \sum_{i=1}^{q} q \ln \left(1-x_{i(q) e}^{\alpha} T_{1}^{\alpha}\right) \\
& +\sum_{e=1}^{s_{x}} \sum_{i=q+1}^{n}\left[(\alpha(q+1)-1) \ln \left(x_{i(q+1) e} T_{2}\right)+\ln \left(1-x_{i(q+1) e}\right)\right]+\sum_{e=1}^{s_{x}} \sum_{i=q+1}^{n}(q-1) \ln \left(1-x_{i(q+1) e}^{\alpha} T_{2}^{\alpha}\right) \\
& \quad+\sum_{h=1}^{s_{y}} \sum_{j=1}^{m}(\beta k-1) \ln \left[y_{j(k) h}\left(2-y_{j(k) h}\right)\right]+\sum_{h=1}^{s_{y}} \sum_{j=1}^{m} \ln \left(1-y_{j(k) h}\right)+(k-1) \ln \left(1-y_{j(k) h}^{\beta}\left(2-y_{j(k) h}\right)^{\beta}\right) .
\end{aligned}
$$

The ML estimators of both parameters are determined by maximizing $\ln \ell_{5}$. The first partial derivatives of $\alpha$ and $\beta$ are represented by

$$
\begin{aligned}
\frac{\partial \ln \ell_{5}}{\partial \alpha}= & \frac{n^{*}}{\alpha}+\sum_{e=1}^{s_{x}} \sum_{i=1}^{q} q \ln \left(x_{i(q) e} T_{1}\right)+\sum_{e=1}^{s_{x}} \sum_{i=q+1}^{n}(q+1) \ln \left(x_{i(q+1) e} T_{2}\right)-\sum_{e=1}^{s_{x}} \sum_{i=1}^{q} \frac{q \ln \left(x_{i(q) e} T_{1}\right)}{x_{i(q) e}^{-\alpha} T_{1}^{-\alpha}-1} \\
& +\sum_{e=1}^{s_{x}} \sum_{i=q+1}^{n} \frac{(q-1) \ln \left(x_{i(q+1) e} T_{2}\right)}{x_{i(q+1) e}^{-\alpha} T_{2}^{-\alpha}-1} \\
\frac{\partial \ln \ell_{5}}{\partial \beta}= & \frac{m^{*}}{\beta}+\sum_{h=1}^{s_{y}} \sum_{j=1}^{m} k \ln \left[y_{j(k) h}\left(2-y_{j(k) h}\right)\right]-\sum_{h=1}^{s_{y}} \sum_{j=1}^{m} \frac{(k-1) \ln \left[y_{j(k) h}\left(2-y_{j(k) h}\right)\right]}{y_{j(k) h}^{-\beta}\left(2-y_{j(k) h}\right)^{-\beta}-1} .
\end{aligned}
$$

The ML estimators of the parameters are constructed by setting Equations (24) and (25) to zero and solving them numerically. Putting the ML estimator of $\alpha$ and $\beta$ in Equation (2), we obtain the SS reliability estimator.

\section{Simulation Results}

The results of a simulation are compared with the performance of estimators based on RSS and MRSS. For a wide range of sample sizes and parameter settings, the absolute 


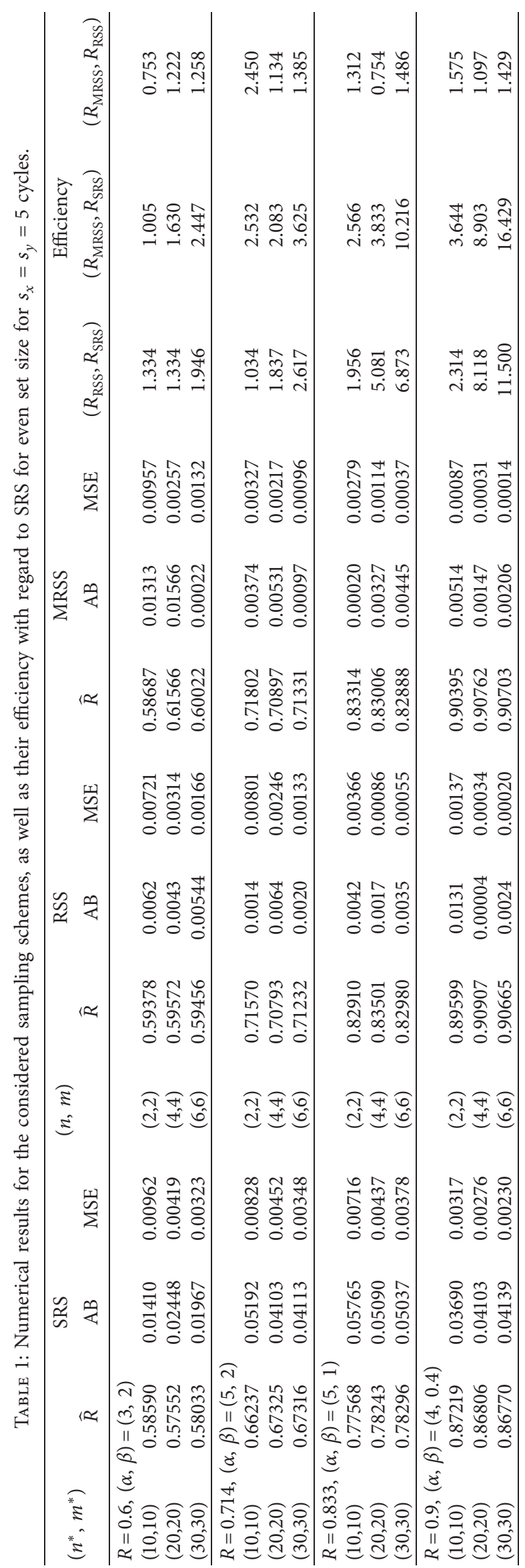




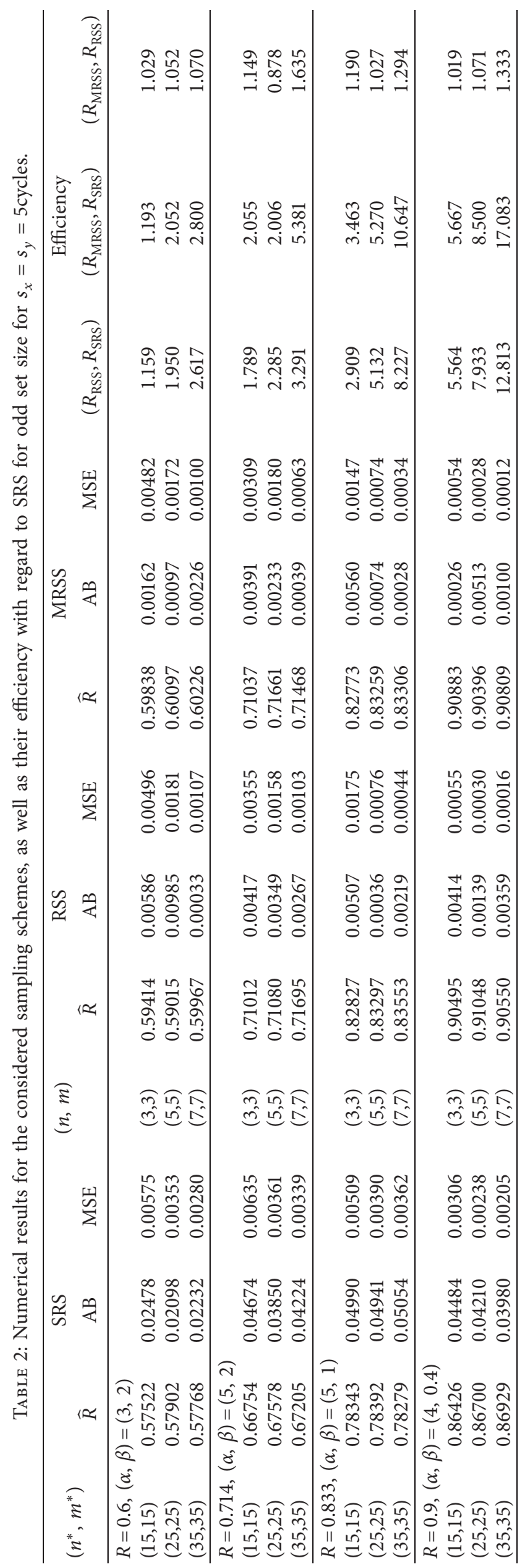




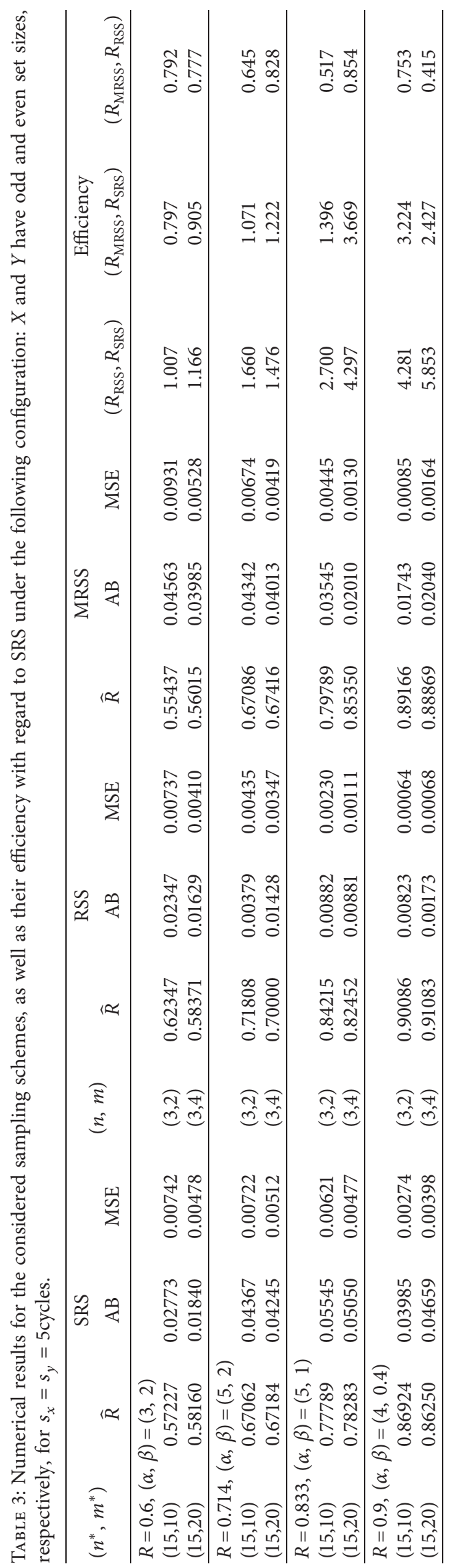




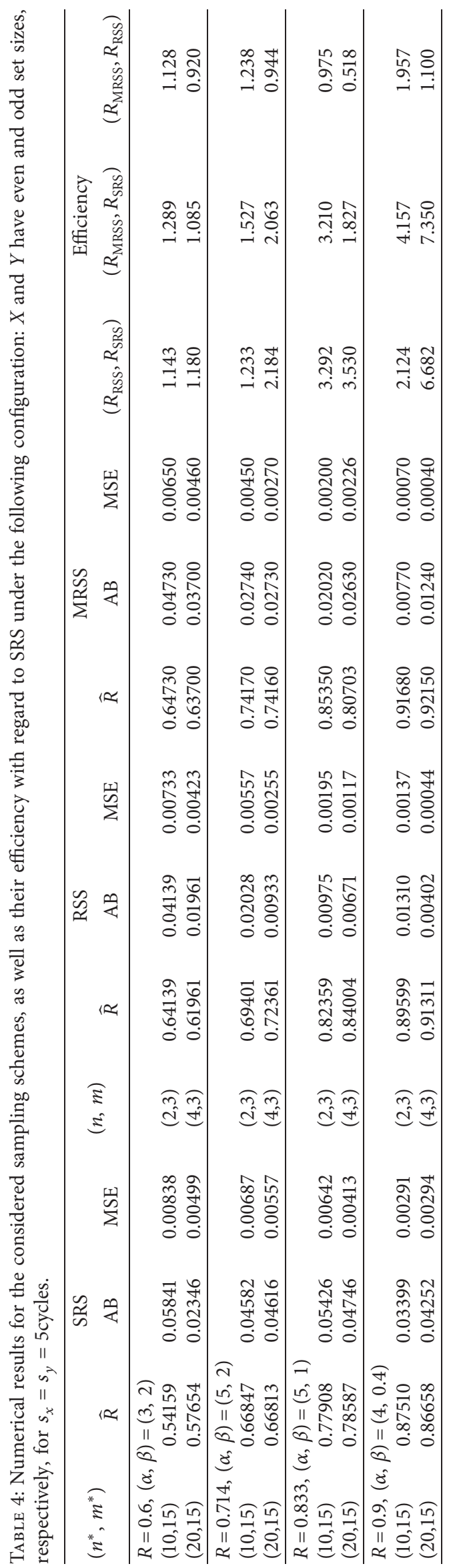


bias (AB), mean square error (MSE), and relative efficiency (RE) criteria are used to investigate the estimated reliability. The following set sizes and number of cycles are taken into account when developing the simulation: $(n, m)=(2,2),(2$, $3),(3,2),(3,3),(3,4),(4,3),(4,4),(5,5),(6,6),(7,7)$, and $s_{x}=s_{y}=5$, respectively. As presented earlier, the sample sizes are given by $s_{x} n$ and $s_{y} m$ for the RSS and MRSS sampling schemes. Also, $\left(n^{*}, m^{*}\right)=(10,10),(10,15),(15,10),(15,15)$, $(15,20),(20,15),(20,20),(25,25),(30,30),(35,35)$ are selected as sample sizes for SRS. The parameter values are determined as $(\alpha, \beta)=(3,2),(5,2),(5,1),(4,0.4)$, giving $R=0.600,0.714,0.833$, and 0.933 , respectively. From the distributions $\operatorname{TL}(\alpha)$ and $\operatorname{TL}(\beta), 1000$ random samples are generated. In this setting, the definition of efficiency is as follows:

$$
\begin{aligned}
\operatorname{efficiency}\left(R_{\mathrm{RSS}}, R_{\mathrm{SRS}}\right) & =\frac{\operatorname{MSE}\left(R_{\mathrm{SRS}}\right)}{\operatorname{MSE}\left(R_{\mathrm{RSS}}\right)}, \\
\operatorname{efficiency}\left(R_{\mathrm{MRSS}}, R_{\mathrm{SRS}}\right) & =\frac{\operatorname{MSE}\left(R_{\mathrm{SRS}}\right)}{\operatorname{MSE}\left(R_{\mathrm{MRSS}}\right)}, \\
\text { efficiency }\left(R_{\mathrm{MRSS}}, R_{\mathrm{RSS}}\right) & =\frac{\operatorname{MSE}\left(R_{\mathrm{RSS}}\right)}{\operatorname{MSE}\left(R_{\mathrm{MRSS}}\right)} .
\end{aligned}
$$

Furthermore, the absolute bias is defined $\operatorname{byAB}\left(R_{\delta}\right)=\left|R-E\left(R_{\delta}\right)\right|, \delta=$ SRS, RSS, MRSS.

The ABs and MSEs of the reliability estimates based on the SRS, RSS, and MRSS are summarized in Tables 1-4. They also show the efficiency of the RSS and MRSS-based reliability estimates with respect to the SRS, as well as the efficiency of the MRSS with respect to the RSS, for different sample sizes and distribution parameters.

We can deduce the following from Tables 1-4:

(i) For all scenarios, the reliability estimates under the RSS scheme outperform the corresponding ones under the SRS (see Tables 1-4)

(ii) The reliability estimates under the MRSS scheme outperform the corresponding ones under SRS in most situations, except when $X$ has an odd set size and $Y$ has even set sizes at $R=0.6$ (see Tables $1-4$ )

(iii) The reliability estimates under the MRSS scheme outperform the corresponding ones under the RSS in most situations, except at $(n, m)=(2,2)$ where $R=0.6$ and at $(n, m)=(4,4)$ where $R=0.833$ (see Table 1)

(iv) The reliability estimates under the MRSS scheme outperform than the corresponding ones under the RSS in most situations, except at $(n, m)=(5,5)$, where $R=0.714$ (see Table 2)

(v) The reliability estimates under the RSS scheme outperform the corresponding ones under the MRSS for all cases (see Table 3)

(vi) The reliability estimates under the RSS scheme outperform the corresponding ones under the MRSS for all cases for $(n, m)=(2,3)$ except at $R=0.833$ while the reliability estimates under the
MRSS scheme outperform the corresponding ones under the RSS for all of the situations at $(n, m)=$ (4,3) except $R=0.9$ (see Table 4 )

\section{Conclusions}

In this manuscript, we have examined the estimation of the unknown reliability measure $R=P[Y<X]$, assuming that $X$ and $Y$ are modeled by independent identically distributed RVs from the TL distribution. We obtain the ML estimator of $R$ in the setting of the SRS or RSS. In the MRSS design, we establish the reliability estimator of $R$ in four situations. In the first and second situations, we obtain the reliability estimator when both $X$ and $Y$ have the same set size. The reliability estimator is derived in the third and fourth situations when the observed samples from the stress distribution have the MRSSO and the observed samples from the strength distribution have the MRSSE, and vice versa. We check the performance of different estimates through numerical studies.

\section{Data Availability}

The data are fully included in the article or the mentioned references.

\section{Conflicts of Interest}

The authors declare that there are no conflicts of interest.

\section{Authors' Contributions}

All authors have contributed equally to this work, and they read and agreed to the published version of the manuscript.

\section{Acknowledgments}

This project was funded by the Deanship of Scientific Research (DSR), at King Abdulaziz University, Jeddah, under grant no. KEP-PhD-69-130-42. The authors, therefore, acknowledge with thanks to DSR technical and financial support.

\section{References}

[1] S. Nadarajah and S. Kotz, "Moments of some J-shaped distributions," Journal of Applied Statistics, vol. 30, no. 3, pp. 311-317, 2003.

[2] S. Kotz and J. R. Van Dorp, Beyond Beta: Other Continuous Families of Distributions with Bounded Support and Applications, World Scientific, Singapore, 2004.

[3] M. E. Ghitany, S. Kotz, and M. Xie, "On some reliability measures and their stochastic orderings for the Topp-Leone distribution," Journal of Applied Statistics, vol. 32, no. 7, pp. 715-722, 2005.

[4] S. Nadarajah and S. Kotz, "The beta exponential distribution," Reliability Engineering \& System Safety, vol. 91, no. 6, pp. 689-697, 2006.

[5] D. Vicari, J. R. Van Dorp, and S. Kotz, "Two-sided generalized Topp and leone (TS-GTL) distributions," Journal of Applied Statistics, vol. 35, no. 10, pp. 1115-1129, 2008. 
[6] A. İ. Genç, "Estimation of $\mathrm{P}(\mathrm{X}>\mathrm{Y})$ with topp-leone distribution," Journal of Statistical Computation and Simulation, vol. 83, no. 2, pp. 326-339, 2013.

[7] S. M. T. K. MirMostafaee, "On the moments of order statistics coming from the Topp-Leone distribution," Statistics \& Probability Letters, vol. 95, pp. 85-91, 2014.

[8] S. M. T. K. MirMostafaee, M. Mahdizadeh, and M. Aminzadeh, "Bayesian inference for the Topp-Leone distribution based on lower k-record values," Japan Journal of Industrial and Applied Mathematics, vol. 33, no. 3, pp. 637669, 2016.

[9] H. A. Bayoud, "Admissible minimax estimators for the shape parameter of Topp-Leone distribution," Communications in Statistics - Theory and Methods, vol. 45, no. 1, pp. 71-82, 2016.

[10] G. McIntyre, "A method for unbiased selective sampling, using ranked sets," Australian Journal of Agricultural Research, vol. 3, no. 4, pp. 385-390, 1952.

[11] K. Takahasi and K. Wakimoto, "On unbiased estimates of the population mean based on the sample stratified by means of ordering," Annals of the Institute of Statistical Mathematics, vol. 20, no. 1, pp. 1-31, 1968.

[12] T. R. Dell and J. L. Clutter, "Ranked set sampling theory with order statistics background," Biometrics, vol. 28, no. 2, pp. 545-555, 1972.

[13] H. A. Muttlak, "Median ranked set sampling," Journal of Applied Statistical Science, vol. 6, pp. 245-255, 1997.

[14] R. A. Johnson, "3 Stress-strength models for reliability," Handbook of Statistics, vol. 7, pp. 27-54, 1988.

[15] S. Kotz, Y. Lumelskii, and M. Pensky, The Stress-Strength Model and its Generalizations: Theory and Applications, World Scientific, Singapore, 2003.

[16] S. Sengupta and S. Mukhuti, "Unbiased estimation ofP(X > Y) for exponential populations using order statistics with application in ranked set sampling," Communications in Statistics - Theory and Methods, vol. 37, no. 6, pp. 898-916, 2008.

[17] H. A. Muttlak, W. A. Abu-Dayyeh, M. F. Saleh, and E. AlSawi, "Estimating $\mathrm{P}(\mathrm{Y}<\mathrm{X})$ using ranked set sampling in case of the exponential distribution," Communications in Statistics - Theory and Methods, vol. 39, no. 10, pp. 1855-1868, 2010.

[18] A. S. Hassan, S. Assar, and M. Yahya, "Estimation of R= P $[\mathrm{Y}<\mathrm{X}]$ for Burr type XII distribution based on ranked set sampling," International Journal of Basic and Applied Sciences, vol. 3, no. 3, pp. 274-280, 2014.

[19] A. S. Hassan, S. Assar, and M. Yahya, "Estimation of $\mathrm{P}(\mathrm{Y}<\mathrm{X})$ for Burr distribution under several modifications for ranked set sampling," Australian Journal of Basic and Applied Sciences, vol. 9, no. 1, pp. 124-140, 2015.

[20] F. G. Akgül and B. Şenoğlu, "Estimation of $\mathrm{P}(\mathrm{X}<\mathrm{Y})$ using ranked set sampling for the Weibull distribution," Quality Technology \& Quantitative Management, vol. 14, no. 3, pp. 296-309, 2017.

[21] F. G. Akgül, Ș. Acıtaş, and B. Şenoğlu, "Inferences on stressstrength reliability based on ranked set sampling data in case of Lindley distribution," Journal of Statistical Computation and Simulation, vol. 88, no. 15, pp. 3018-3032, 2018.

[22] A. Ibrahim Al-Omari, I. M. Almanjahie, A. S. Hassan, and H. F. Nagy, "Estimation of the stress-strength reliability for exponentiated Pareto distribution using median and ranked set sampling methods," Computers, Materials \& Continua, vol. 64 , no. 2, pp. 835-857, 2020.

[23] A. S. Hassan, A. Al-Omari, and H. F. Nagy, "Stress-strength reliability for the generalized inverted exponential distribution using MRSS," Iranian Journal of Science and Technology Transaction A-Science, vol. 45, no. 2, pp. 641-659, 2021. 